\title{
Isospin Breaking in the Extraction of Isovector and Isoscalar Spectral Functions from $e^{+} e^{-} \rightarrow$ hadrons
}

\author{
K. Maltman* and C.E. Wolfe ${ }^{\dagger}$ \\ ${ }^{*}$ Dept. Mathematics and Statistics, York Univ., 4700 Keele St., Toronto, ON Canada, and \\ CSSM, Univ. of Adelaide, Adelaide, SA Australia ${ }^{1}$ \\ ${ }^{\dagger}$ Nuclear Theory Center, Indiana University, Bloomington, IN, USA
}

\begin{abstract}
A finite energy sum rule (FESR) analysis of the isospin-breaking vector current correlator $\left\langle 0\left|T\left(V_{\mu}^{3} V_{\nu}^{8}\right)\right| 0\right\rangle$ is used to determine the isospin-breaking electromagnetic (EM) decay constants of the low-lying vector mesons. These results are used to evaluate the corrections required to extract the flavor diagonal 33 and 88 resonance contributions from the full resonance EM contributions to the EM spectral function. A large $(\sim 15 \%)$ correction is found in the case of the $\omega$ contribution to the isoscalar spectral function. The implications of these results for sum rules based on the isovector-isoscalar spectral difference are considered.
\end{abstract}

\section{INTRODUCTION}

If we define the flavor $a b(a, b=3,8)$ vector current correlators via

$$
i \int d^{4} x e^{i q x}\left\langle 0\left|T\left(V_{\mu}^{a}(x) V_{\nu}^{b}(0)^{\dagger}\right)\right| 0\right\rangle \equiv\left(-g_{\mu \nu} q^{2}+q_{\mu} q_{\nu}\right) \Pi^{a b}\left(q^{2}\right),
$$

the corresponding EM current-current correlator is given by

$$
\Pi^{E M}=\Pi^{33}+\frac{2}{\sqrt{3}} \Pi^{38}+\frac{1}{3} \Pi^{88},
$$

which reduces to $\Pi^{33}+\Pi^{88} / 3$ in the isospin limit. $\Pi^{E M}$ is related to the hadronic electroproduction cross-section by

$$
\sigma\left(e^{+} e^{-} \rightarrow \text { hadrons }\right)=\frac{16 \pi^{2} \alpha^{2}}{s} \operatorname{Im} \Pi^{E M}(s) .
$$

In the isospin limit, the 33 and 88 contributions associated with $n \pi$ states can be classified by $G$-parity, making an experimental determination of the separate

1) Supported by the Natural Sciences and Research Engineering Council of Canada 
isovector (33) and isoscalar (88) components of the EM spectral function, $\rho^{E M}(s)$, possible. Isospin breaking (IB), however, complicates this separation because of the presence of a non-zero 38 spectral component. One contribution to the 38 component, associated with the process $e^{+} e^{-} \rightarrow \omega \rightarrow \pi^{+} \pi^{-}$, is conventionally removed by hand in determining the 33 spectral function, but other 38 contributions also exist which are not so subtracted. If we define, in obvious notation, the isovector (3) and isoscalar (8) components of the EM decay constant of vector meson, $V$, via

$$
F_{V}^{E M}=F_{V}^{3}+\frac{1}{\sqrt{3}} F_{V}^{8}
$$

then, for example, the intermediate $\rho$ contribution to $\rho^{E M}(s)$ contains not only a 33 component, proportional to $\left[F_{\rho}^{3}\right]^{2}$, but also, to leading order in IB, a 38 component proportional to $F_{\rho}^{3} F_{\rho}^{8}$. The $\omega$ contribution to $\rho^{E M}(s)$ similarly contains a 38 component proportional to $F_{\omega}^{3} F_{\omega}^{8}$.

The presence of these 38 "contaminations" in the conventionally extracted 33 and 88 spectral functions creates potential problems for

- $\mathrm{CVC}$ tests

- the determination of $m_{s}$ using Narison's 33 - 88 "tau-decay-like" sum rule [1]

- The inverse weighted chiral $33-88$ sum rule for the $6^{\text {th }}$ order ChPT LEC, $Q_{V}$, which governs flavor/isospin breaking in vector current correlators [2].

In the latter two cases, the IB effect can be particularly important (1) because the cancellation between the nominal isovector and isoscalar integrals is typically rather strong (to the $\sim 10 \%$ level) and, (2) because the 38 component of the $\rho$ and $\omega$ EM decay constants induced by $\rho-\omega$ mixing has the opposite sign for the $\rho$ and $\omega$, the 38 corrections add constructively when one forms the nominal isovector-isoscalar difference.

It is easy to see that the relative importance of this effect should be much larger for the isovector "contamination" of the $\omega$ decay constant than for the isoscalar "contamination" of the $\rho$ decay constant. Indeed, taking $S U(3)_{F}$ for the vector current vacuum-to-vector-meson matrix elements, and assuming ideal mixing of the vector meson sector, $F_{\rho}^{(0)} \simeq 3 F_{\omega}^{(0)}$, where the superscript $(0)$ indicates the isospin-unmixed states. With $\epsilon$ the $\rho-\omega$ mixing angle, one then has

$$
\begin{aligned}
& F_{\rho}^{E M}=F_{\rho}^{(0)}-\epsilon F_{\omega}^{(0)} \simeq F_{\rho}^{(0)}\left(1-\frac{\epsilon}{3}\right) \\
& F_{\omega}^{E M}=F_{\omega}^{(0)}+\epsilon F_{\rho}^{(0)} \simeq F_{\omega}^{(0)}(1+3 \epsilon) .
\end{aligned}
$$

The fractional contribution of the "wrong-isospin" current to the EM decay constant of the $\omega$ should thus be 9 times that of the corresponding IB contribution to $F_{\rho}^{E M}$, in the limit that the effect is dominated by $\rho-\omega$ mixing. 
In this paper, we employ a FESR analysis of the IB vector current correlator, $\Pi^{38}$, in order to extract the product, $F_{V}^{3} F_{V}^{8}$, of IB and isospin-conserving (IC) decay constants for the low-lying vector mesons. This allows us to determine the 38 contamination of the resonance contributions to the nominally extracted 33 and 88 spectral functions, and hence to make the corrections required to extract the actual versions thereof from data.

\section{FESR'S AND THE DETERMINATION OF THE IB VECTOR MESON DECAY CONSTANTS}

The analyticity structure of $\Pi^{38}$ is such that, for any function $w(s)$ analytic in the region of the contour, one has, using Cauchy's theorem, the FESR relation

$$
\int_{s_{t h}}^{s_{0}} d s w(s) \rho^{38}(s)=\frac{-1}{2 \pi i} \oint_{|s|=s_{0}} d s w(s) \Pi^{38}(s),
$$

where $\rho^{38}(s)=\frac{1}{\pi} \operatorname{Im} \Pi^{38}(s)$. Choosing $s_{0}$ large enough that $\Pi^{38}$ can be represented by the OPE on the RHS, but small enough that $\rho^{38}$ on the LHS is still dominated by the known isovector and isoscalar vector mesons, one obtains a relation between the products $f_{V}=F_{V}^{3} F_{V}^{8}$ which govern the sizes of resonance contributions to $\rho^{38}$ and the parameters ( $\alpha_{s}$ and vacuum condensates) entering the OPE. Since the OPE is well known this allows us to constrain the resonance parameters.

In order to make the analysis suggested above reliable, we will employ a form of FESR tested in the isovector vector channel and shown to be very accurately satisfied there [3]. These "pinch-weighted" FESR's are those corresponding to weights satisfying $w\left(s_{0}\right)=0$. In the isovector vector channel, where they can be tested, they are known to be very accurately satisfied down to rather low scales $\left(\sim 2 \mathrm{GeV}^{2}\right.$, even when more general FESR's, which do not suppress contributions from the region near the timelike real axis, are poorly satisfied [3]). It is convenient to work with two families of pinched weights, $w_{s}(s)=\left[1-s / s_{0}\right]\left[1+A s / s_{0}\right]$ and $w_{d}(s)=\left[1-s / s_{0}\right]^{2}\left[1+A s / s_{0}\right]$, where in each case $A$ is a free parameter, used to vary the weight profile. It is worth noting that, using the FESR's resulting from these two weight families to fit the decay constants of the first three $\rho$ resonances, one obtains a determination of the $\rho(770)$ decay constant in terms of OPE parameters which is accurate to within experimental errors $[3,4]$.

On the hadronic side of our FESR's we assume a sum of Breit-Wigner resonance contributions for the $\rho, \omega$, and $\phi$ with PDG values of the masses and widths. In the $\rho^{\prime}-\omega^{\prime}$ region, a single effective resonance contribution with the average values of the masses and widths is employed since the individual $\rho^{\prime}$ and $\omega^{\prime}$ masses and widths are very similar, making it impossible for our sum rule to discriminate between them. The necessity of including a possible $\phi$ term has been discussed in Ref. [5], while the importance of including the resonance widths has been stressed in Ref. [6]). The only remaining unknowns in the spectral ansatz are the products $f_{V}=F_{V}^{3} F_{V}^{8}$, which determine the heights of the resonance peaks. 
The $D=0,2,4,6$ terms in the OPE representation of $\Pi^{38}$ are given by

$$
\begin{aligned}
{\left[\Pi_{1 \gamma E}^{38}\right]_{D=0}=} & -\frac{\alpha}{16 \pi^{3}} \frac{1}{4 \sqrt{3}} \ln \left(Q^{2}\right) \\
{\left[\Pi^{38}\left(Q^{2}\right)\right]_{D=2}=} & \frac{3}{2 \pi^{2} Q^{2}} \frac{1}{4 \sqrt{3}}\left[\left(m_{d}^{2}-m_{u}^{2}\right)\left(Q^{2}\right)\right]\left[1+\frac{8}{3} a\left(Q^{2}\right)\right. \\
& \left.+\left(\frac{17981}{432}+\frac{62}{27} \zeta(3)-\frac{1045}{54} \zeta(5)\right) a^{2}\left(Q^{2}\right)\right] \\
{\left[\Pi^{38}\left(Q^{2}\right)\right]_{D=4}=} & \frac{2\left(\left\langle m_{u} \bar{u} u\right\rangle-\left\langle m_{d} \bar{d} d\right\rangle\right)}{4 \sqrt{3} Q^{4}}\left[1+\frac{1}{3} a\left(Q^{2}\right)+\frac{11}{2} a^{2}\left(Q^{2}\right)\right] \\
{\left[\Pi^{38}\left(Q^{2}\right)\right]_{D=6}=} & \frac{112 \pi}{81 \sqrt{3} Q^{6}} \rho_{\text {red }} \gamma\left(\rho \alpha_{s}\langle\bar{q} q\rangle^{2}\right)
\end{aligned}
$$

where $\alpha$ is the usual EM coupling, $a\left(Q^{2}\right)=\alpha_{s}\left(Q^{2}\right) / \pi, \zeta(n)$ is the Riemann zeta function, $\gamma=[<\bar{d} d>/<\bar{u} u>]-1$, and $\rho_{\text {red }}$ is the ratio of the violation of the vacuum saturation approximation (VSA) estimate for the $D=6$ condensate combination in the 38 channel to that in the flavor-diagonal 33 vector channel (see Ref. [8] for the latter value). Numerically, the $D=4$ term is the largest, owing to the smallness of $\alpha$ and $m_{u, d}$. It can be rewritten in terms of the IB mass ratio $r \equiv\left(m_{d}-m_{u}\right) /\left(m_{d}+m_{u}\right)$ (for which we use the value from Leutwyler's ChPT analysis [7]) and $<\left(m_{u}+m_{d}\right) \bar{u} u>\simeq-f_{\pi}^{2} m_{\pi}^{2}$ (where we have used the GMOR relation). We employ the 4-loop versions of the running mass and coupling.

Lack of knowledge of $\rho_{\text {red }}$ would normally limit the accuracy of the extraction of the $f_{V}$. Using both the $w_{s}$ and $w_{d}$ families of FESR's, however, it turns out to be possible to determine $\rho_{\text {red }}$ self-consistently. In Figure 1 we illustrate the dependence of $f_{\rho}$ on $\rho_{\text {red }}$ for both the $w_{s}$ and $w_{d}$ weight families. Obviously only one value of $\rho_{\text {red }}$ provides a consistent determination of $f_{\rho}$. Checking analogous figures for the other $f_{V}$, one finds that the values of $\rho_{\text {red }}$ for which they become consistent agree with that in Figure 1 to better than 1\%. This clearly demonstrates that this "selfconsistency" determination of $\rho_{\text {red }}$ is physically meaningful. It is interesting to note that the degree of VSA violation is very similar for the flavor-diagonal isovector and IB flavor 38 correlators. Further details of the calculation, including OPE input values, uncertainties, and a description of the contour-improved implementation of the OPE integrals [9], may be found in Ref. [10].

Having extracted $f_{V}$, and knowing the EM decay constants, $F_{V}^{E M}$, one may then separately determine $F_{V}^{3}$ and $F_{V}^{8}$ for $V=\rho, \omega, \phi$. To understand the impact of the IB decay constants on the extraction of the 33 and 88 spectral functions, it is convenient to quote the results in terms of the squared ratios, $r_{V}$, shown below. In each case, the numerator in the ratio of decay constants represents the IC decay constant of the meson in question (whose square is relevant to the contribution to either the 33 or 88 spectral function), while the denominator represents the nominal value of the numerator, obtained from EM data neglecting the presence of the "wrong-isospin" IB contributions discussed above. The deviations of the $r_{V}$ 
from 1 reflect the presence of the IB $F_{\rho}^{8}, F_{\omega}^{3}$ and $F_{\phi}^{3}$ contributions to $F_{\rho, \omega, \phi}^{E M}$. The ratios have been defined in such a way that the true resonance contributions to the 33 (or 88) spectral functions are obtained by multiplying the nominal results, obtained in the usual analysis, by $r_{V}$. The results of the analysis outlined above are then

$$
\begin{aligned}
& r_{\rho}=\left[\frac{F_{\rho}^{3}}{F_{\rho}^{E M}}\right]^{2}=0.982 \pm 0.0021 \\
& r_{\omega}=\left[\frac{F_{\omega}^{8}}{\sqrt{3} F_{\omega}^{E M}}\right]^{2}=1.154 \pm 0.017 \\
& r_{\phi}=\left[\frac{F_{\phi}^{8}}{\sqrt{3} F_{\phi}^{E M}}\right]^{2}=1.009 \pm 0.001,
\end{aligned}
$$

where the errors are dominated by the uncertainty in the quark mass ratio, $r$, and hence are strongly correlated.

We note the following features of the above results:

- The effect on the $\rho$ contribution to the 33 spectral function is small, and hence has no impact on CVC tests, given the current experimental errors on the EM cross-sections.

- As expected from the argument above, the effect is much larger for the $\omega$ contribution to the 88 spectral function. Our result corresponds to a $\sim 7.5 \%$ isovector contribution to the physical $\omega$ decay constant.

- The ratio of $\rho$ and $\omega$ corrections is $8.6 \simeq 9$, suggesting dominance by $\rho$ - $\omega$ mixing.

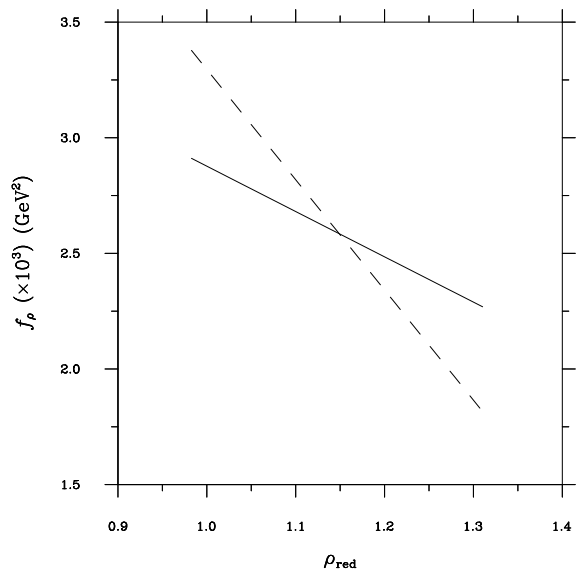

FIGURE 1. Dependence of the $\rho$ spectral parameter, $f_{\rho}$, on the reduced VSA-violation parameter, $\rho_{\text {red }}$, for the two weight families, $w_{s}$ and $w_{d}$. The solid line corresponds to $w_{s}$, the dashed line to $w_{d}$. 
The impact of these corrections on the 33-88 sum rules discussed above is as follows.

- Using $\tau$ decay data for the isovector part, and the above corrections for the EM isoscalar data, the Narison $m_{s}$ sum rule implies

$$
m_{s}\left(1 \mathrm{GeV}^{2}\right)=154( \pm \sim 50) \mathrm{MeV}
$$

compatible with value $157 \pm 16 \pm 15 \pm 16$ obtained in a recent analysis based on flavor breaking in hadronic $\tau$ decays [11], and to be compared to the central value $197 \mathrm{MeV}$ obtained neglecting the presence of IB corrections [1].

- The correction to the inverse chiral $33-88$ sum rule extraction of the $6^{\text {th }}$ order ChPT low-energy constant $Q_{V}$ is also large. The FESR solution above implies, via an inverse moment 38 sum rule, the value [10]

$$
Q_{V}\left(m_{\rho}^{2}\right)=(3.3 \pm 0.4) \times 10^{-5},
$$

to be compared to the value from a recent analysis [12] of the vector current $\Pi^{u d}-\Pi^{u s}$ difference, employing $\tau$ decay spectral data (which does not need IB corrections)

$$
Q_{V}\left(m_{\rho}^{2}\right)=(2.8 \pm 1.3) \times 10^{-5}
$$

The agreement of the results of Eq. (10) with the independent determination of Eq. (11) provides further strong support for the values of the IB vector meson decay constants obtained in the present analysis.

\section{REFERENCES}

1. Narison, S., Phys. Lett. B358, 113 (1995).

2. Golowich, E. and Kambor, J., Nucl. Phys. B447, 373 (1995); Phys. Rev. D53, 2651 (1996).

3. Maltman, K., Phys. Lett. B440, 367 (1998).

4. Maltman, K., Phys. Lett. B467, 14 (1999).

5. Maltman, K., Phys. Rev. D53, 2563 (1996).

6. Iqbal, M.J., Jin, X-M. and Leinweber, D.B., Phys. Lett. B367, 45 (1996).

7. Leutwyler, H., Phys. Lett. B374, 163 (1996); Phys. Lett. B378, 313 (1996).

8. Narison, S. Phys. Lett. B361, 121 (1995).

9. Le Diberder, F. and Pich, A., Phys. Lett. B286, 147 (1992); B289, 165 (1992).

10. Maltman, K. and Wolfe, C.E., Phys. Rev. D59, 096003 (1999).

11. Kambor, J. and Maltman, K., "The Strange Quark Mass From Flavor Breaking in Hadronic $\tau$ Decays", in preparation.

12. Dürr, S. and Kambor, J., hep-ph/9907539. 